\title{
Multi-university Internet video-conferencing course provides novel approach to student-directed learning
}

\author{
AdAm DURAnt and WILlam RoSE, Michigan Technological University, Houghton, USA \\ Crystal Mann and John Stix, McGill University, Montreal, Canada
}

Connecting graduate students with experts in a given discipline is vital to their success but has often been challenging because of logistics, costs, and scheduling. However, advances in Internet video conferencing now allow multiple parties to meet simultaneously and engage in real-time discussion.

This new technology allowed us to design a graduate-level seminar in which students were able to directly engage in scientific discussion with experts on volcano instability. Conducted under the auspices of the Earth Hazards (EHaz) Consortium, the spring 2007 Volcano Instability course connected nine previously disparate groups in a combined learning and cultural experience, and followed on from a similar class offered last year (Mann et al., 2006; Rose and Stix, 2006). Course participants (including guest speakers, students, and university professors) used Marratech Internet video conferencing software to meet twice weekly with the objective of promoting

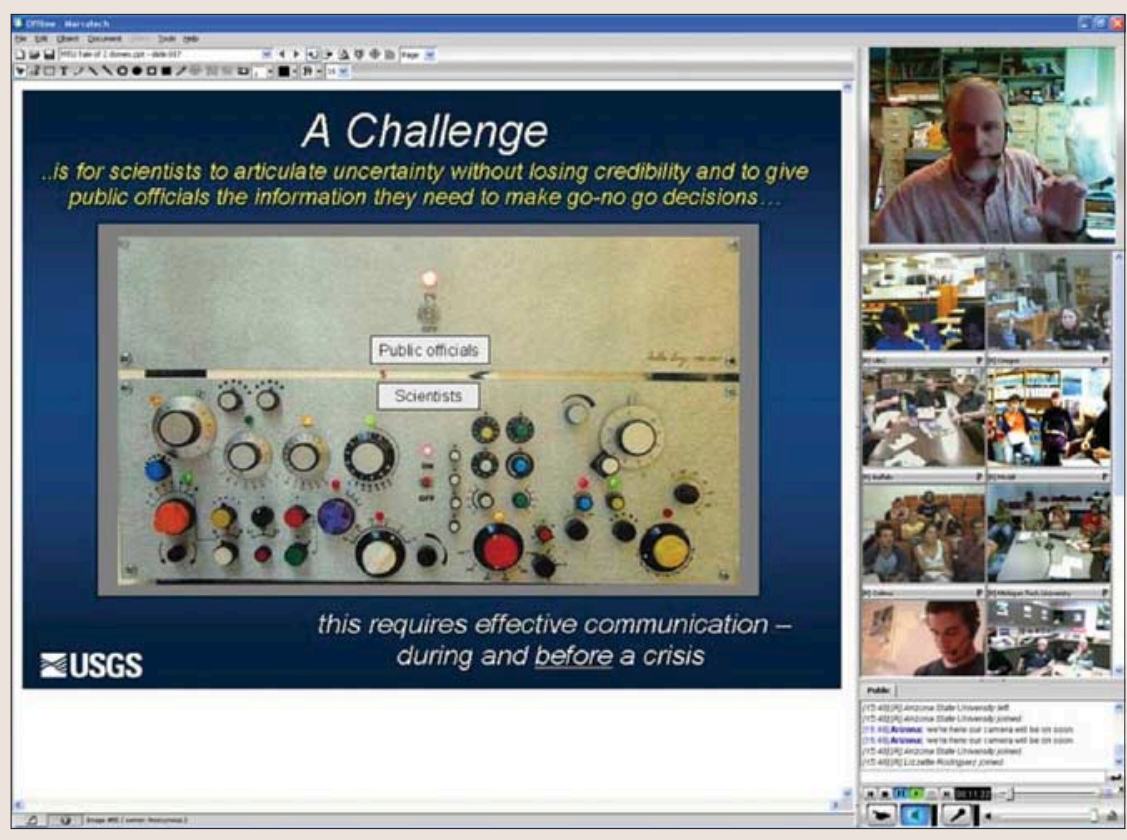

Figure 1. View of the Marratech browser during a class discussion with guest speaker John Pallister. student-directed learning. Many universities can participate through this approach, allowing an advanced graduate class that normally contains $4-8$ students on one campus to become a class of 50 or more dispersed over many campuses.

Course mechanics. The course involved 78 participants (students, professors, and scientists) from nine universities and organizations in Canada, France, Mexico, Russia, U.K., and the U.S. The software provides a multifaceted interactive environment in which participants can view each other and display Microsoft Word, PowerPoint, and Adobe PDF files on a common "white board." Class participants included graduate and undergraduate students. The language spoken during the class was English, although there were two groups whose first language was Spanish and one group whose first language was French. The 14 guest speakers were recognized, cutting-edge scientists at the forefront of research in their field. The 14 guest speakers were recognized, cutting-edge scientists at the forefront of research in their field and included Falk Amelung (University of Miami, USA), Eliza Calder (University at Buffalo, USA), Gerardo Carrasco (Universidad Nacional Autónoma de México, Mexico), Dave Clague (Monterey Bay Aquarium Research Institute, USA), Corey Froese (Alberta Geological Survey, Canada), Jeff Johnson (University of New Hampshire, USA), Oleg Melnik (Lomonosov Moscow State University, Russia), John Pallister (USGS Cascades Volcano Observatory, USA), Mike Ramsey (University of Pittsburgh, USA), Paul Segall (Stanford University, USA), Lee Siebert

(Editor's note: This article was previously published in part in the Geological Society of America Geoscience Education Division Newsletter, Summer 2007).
(Smithsonian Global Volcanism Program, USA), Hugh Tuffen (Lancaster University, U.K.), Sylvie Vergniolle (Institut de physique du globe de Paris, France), and Benjamin van Wyk de Vries (OPGC de Clermont-Ferrand, France).

The Volcano Instability course Web site served as the medium for the transfer of core information required for the class. At least one week before the first weekly meeting, a selection of articles and an electronic presentation were provided by the guest speaker and posted on the Web site. The students were then tasked with reviewing the materials prior to the discussion sessions. Additionally, after each meeting, recordings were posted to allow participants to review the discussion. The material presented was part of new research work and was often still in press or review. For some groups with limited library resources, the Web site provided a unique resource of current research articles and ideas that were vital for ongoing graduate research activities.

Students were charged with the overall moderation of the discussions and each week one university group was assigned with the role of "moderator." This approach directly engaged students in scientific discussion and encouraged social interaction. Prior to the first weekly meeting, students at each university were encouraged to meet as a group to generate basic questions and identify themes for discussion. The first weekly meeting was held on a Tuesday for 60 minutes and included all multi-university students and professors. The objectives were to: (1) discuss background related to the presentation; (2) develop themes related to the presentation; and (3) focus and prioritize important questions for the speaker during the second meeting. The second weekly meeting was held on a Thursday for 90 minutes and included all EHaz class participants, professors, and the guest speaker. This session aimed to have a student-lead sci- 
entific discussion with the guest speaker. The objective was to have a student-lead scientific discussion with the guest speaker. Following a brief introduction at the beginning of the second meeting, the moderator invited each university group to present a question to the speaker. Questions were displayed on the white board to ease language barriers, and a student from a given group posed the question to the class. The speaker would then reply and lead the class to relevant graphics in the presentation (shared through the browser, Figure 1). Following the speaker's reply, the moderator invited follow-up questions from all participants.

Summary. The EHaz Volcano Instability course was an inexpensive and innovative approach to teaching across international boundaries. Using video-conferencing technology and the Internet, students and experts can be connected in a virtual classroom environment.

Until recently, students had the opportunity to interact with experts in a given field on only a few (often serendipitous) occasions. Geographical isolation can mean that experts rarely travel to some academic institutions, denying students the opportunity of direct exposure. This course, however, brought 14 experts directly to 64 students and professors.

As evidenced by overwhelmingly positive course evaluations, the course was a success and students responded that the approach was stimulating and conducive to learning. In addition, participants were able to interact with other groups all over North America, which provided cultural enlightenment and professional understanding.

The technology now exists to provide all tools one would find in a classroom, so there is potential to teach classes interactively. The approach has great potential for application over many disciplines and this dynamic interaction will ultimately raise the standard of instruction and motivate students to self-guide their learning experience to levels of greater understanding.

Suggested reading. Detailed articles on the activities of the EHaz program are currently in preparation. More information on the 2007 EHaz multi-university course on Volcano Instability can be found at: www.geo.mtu.edu/EHaz/VolcanoInstability class/index.htm. "Earth hazards consortium: A unique approach to student-centered learning" by Mann et al. (Eos Transactions, AGU 2006 Fall Meeting Supplemental Abstract ED21A-1222). "Experimental multi-university graduate class in volcanology" by Rose and Stix (EOS Transactions, 2006). TLE

Acknowledgments: Many people played a central role in the evolution of the class and we are grateful for their efforts. They include E. Calder, D. Chard, H. Delgado, T. Dominguez, C. Helo, P. Lins, D. Moran, J. Roberge, M. Smith, J. Stevenson, N. Varley, and G. Williams-Jones. The EHaz consortium consists of six research-based universities in the United States (Michigan Technological University, University of Buffalo), Canada (McGill University, Simon Fraser University), and Mexico (Universidad Nacional Autónoma de Mexico, Universidad de Colima). The consortium is funded by the U.S. Department of Education, Human Resources, and Skills Development Canada, and the Secretaria de Educación Pública of Mexico, as part of the North American Free Trade Agreement.

Corresponding author: ajdurant@mtu.edu 